\title{
Ethnologies
}

\section{Les représentations d'une appartenance culturelle en contexte de migration}

\section{Le cas des Bretons migrants au Québec depuis 1950}

\section{Linda Guidroux}

Volume 33, numéro 1, 2011

URI : https://id.erudit.org/iderudit/1007805ar

DOI : https://doi.org/10.7202/1007805ar

Aller au sommaire du numéro

\section{Éditeur(s)}

Association Canadienne d'Ethnologie et de Folklore

ISSN

1481-5974 (imprimé)

1708-0401 (numérique)

Découvrir la revue

Citer cet article

Guidroux, L. (2011). Les représentations d'une appartenance culturelle en contexte de migration : le cas des Bretons migrants au Québec depuis 1950. Ethnologies, 33(1), 259-293. https://doi.org/10.7202/1007805ar
Résumé de l'article

Dans son article, Linda Guidroux cherche à comprendre comment on peut se sentir de quelque part et pourquoi revendiquer cette appartenance culturelle en situation de migration. Ces préoccupations l'ont mené à se questionner sur la déterritorialisation de l'appartenance culturelle, puis sa relocalisation en contexte de migration. Pour cela, elle a décidé de prendre pour étude de cas la migration des Bretons au Québec depuis 1950. En premier lieu, elle propose de découvrir à quel groupe ethnique (breton, français, québécois, canadien ou autre) les migrants bretons du Québec rencontrés font référence (ou ont fait référence pour ceux retournés en Bretagne) pour s'identifier en terre d'accueil et laquelle de ces appartenances ils choisissent pour se présenter à "l'Autre ". En second lieu, elle analyse dans quelle mesure les représentations que ces migrants se font de leur groupe ethnique de référence a influencé leur choix d'appartenir à ce groupe et de repérer les liens qu'ils ont maintenus, construits ou rompus avec celui-ci. Enfin, elle identifie si leur expérience migratoire au Québec a modelé leur choix d'appartenance culturelle.
Ce document est protégé par la loi sur le droit d'auteur. L'utilisation des services d’Érudit (y compris la reproduction) est assujettie à sa politique d'utilisation que vous pouvez consulter en ligne.

https://apropos.erudit.org/fr/usagers/politique-dutilisation/ 


\section{LES REPRÉSENTATIONS D'UNE APPARTENANCE CULTURELLE EN CONTEXTE DE MIGRATION}

Le cas des Bretons migrants au Québec depuis 1950

\section{Linda Guidroux}

Université Laval

«Comment peut-on se sentir de quelque part et pourquoi revendiquer cette appartenance culturelle $^{1}$ en situation de migration $^{2}$ ?». Tel a été le propos de ma recherche doctorale durant

1. Je privilégie l'emploi du terme d'«appartenance culturelle » qui me semble moins banalisé et plus précis que celui d' "identité culturelle » et moins problématique que celui d'«identité ethnique ». Par appartenance culturelle, j'entends le sentiment très fort d'appartenir à une communauté humaine distincte qui ne prétend à aucune supériorité sur les autres communautés humaines, mais à laquelle certains individus tiennent de façon vitale. Sencébé, dans ses travaux, différencie l'appartenance culturelle de l'identité culturelle, mais considère ces deux notions comme complémentaires : "[I]dentité répond à la question du qui suis-je ? et appartenance répond à la question du comment et avec qui suis-je ?»(2004:24). Autrement dit, selon Sencébé, l'identité renvoie davantage à l'aspect individuel qui fait que la personne humaine existe de façon indépendante par elle-même, dans son être spirituel et corporel ; tandis que l'appartenance fait référence à l'aspect social par lequel l'être humain est inévitablement relié à d'autres êtres humains et établit avec eux des liens dans la durée. C'est ce que les Danois appellent la folkelighed (terme conservé au siècle dernier grâce à Grundtvig), autrement dit ce simple mais profond, indéracinable sentiment d'appartenance, leur danité (Texier 2004 : 171).

2. J'utilise le terme de «migration » pour désigner le processus par lequel les Bretons ont choisi d'émigrer de Bretagne et d'immigrer au Québec, même si certains auteurs l'emploient pour décrire une autre réalité, notamment Kim D. Butler qui désigne par «migrations » exclusivement les migrations professionnelles ou saisonnières qui engendrent un va-et-vient perpétuel entre territoire d'origine et territoire d'accueil : «With improved transportation and communication resources in the twentieth century, seasonal migrations have been made possible over increasing distances. [...] worker today may be able to shuttle between the two places several time a year. Institutions and networks 
quatre ans ${ }^{3}$. Mon objectif était de comprendre comment une appartenance culturelle peut être déterritorialisée, puis relocalisée dans un contexte de migration. Pour cela, j'ai décidé de prendre pour étude de cas la migration des Bretons au Québec depuis 1950. J'ai considéré leur migration comme une situation expérimentale au travers de laquelle j'ai tenté d'analyser comment se construit, se déconstruit ou se reconstruit une appartenance culturelle.

Par le biais de cet article, je propose d'exposer d'une part à quel groupe d'individus les migrants bretons du Québec rencontrés font ou ont fait référence ${ }^{4}$ (pour ceux retournés en Bretagne) pour s’identifier en terre d'accueil. En d'autres termes, se sentent-ils Bretons, Français, Québécois, Canadiens ou autres dans leur terre de migration ? Et laquelle de ces appartenances ont-ils choisie pour se présenter à «l'Autre »? D'autre part, il s'agit d'analyser dans quelle mesure les représentations que ces migrants se font de leur groupe de référence a influencé leur choix d'appartenir à ce groupe et de repérer les liens qu'ils ont maintenus, construits ou rompus avec ce groupe. Enfin, il s'agit d'identifier si leur expérience migratoire au Québec a modelé leur choix d'appartenance culturelle. Autrement dit, en terre d'accueil, se sentent-ils appartenir au même groupe d'individus que celui auquel ils adhéraient en terre d'origine et pourquoi leur expérience migratoire a été ou non facteur de changement?

that form as a result of this type of migration » (2001:202). De la même manière, pour désigner ces individus j'utilise le terme de «migrants »

3. De 2006 à 2010, en cotutelle entre la Faculté des études supérieures de l'Université Laval, Québec (Canada) dans le cadre du programme de doctorat en ethnologie des francophones en Amérique du Nord pour l'obtention du grade de Philosophiae doctor (Ph.D.), sous la direction de Laurier Turgeon et la Faculté des Lettres et Sciences humaines de l'Université de Bretagne occidentale, Brest (France) pour l'obtention du grade de Docteur en ethnologie, sous la direction de Jean-François Simon.

4. Plutôt que le terme de "groupe ethnique breton ", j'ai utilisé le terme de «communauté bretonne » ou «groupe de référence » tout au long de ma recherche. Le sens que j'accorde à ce terme de communauté n'est pas péjoratif et lié à l'idée de sectarisme, tel qu'il est généralement entendu en Europe, mais plutôt dans le sens positif de minorité, communauté ethnique, communauté culturelle, tel que défini par le Conseil des relations interculturelles du Québec. Autrement dit, ce que j'entends par communauté culturelle, c'est surtout un groupe d'individus qui partagent les mêmes rêves et les mêmes sentiments, une « communauté affective» comme le définit Appadurai (2001:35). 
Mais avant de répondre à ces questionnements, il me semble important de présenter le cadre méthodologique que j'ai choisi pour mener cette recherche.

\section{Cadre méthodologique}

Choix du corpus : une approche comparative et une ethnographie multisites

Concernant mon corpus, j'ai choisi volontairement d'interroger des Bretons revendiquant leur appartenance culturelle régionale en terre d'accueil. Cependant, pour avoir une idée plus objective et complète de la question et dans une démarche comparative, j'ai aussi tenu compte des témoignages de certains Bretons se considérant «plus Français que Bretons », voire ayant coupé tous contacts avec la Bretagne et ne cherchant pas à se rapprocher de la communauté bretonne du Québec. À ce sujet, Green explique que le migrant, en tant qu'individu, dans son essence même, juxtapose deux sociétés : la société d'origine et la société d'accueil. Il vit une situation impliquant du comparatif. Le migrant est comparé dans son rapprochement à la société hôte tout comme il est mesuré en fonction de son éloignement progressif par rapport à son pays d'origine (Green 2002 : 23-24). La pensée sur les migrations gagne donc à être comparative. Le phénomène migratoire se situe non seulement dans le temps, mais également dans l'espace (Green 2002 : 123). Selon Marcus, la méthode comparative est la plus appropriée pour cerner la dynamique des processus migratoires et, notamment, les phénomènes de délocalisation et de relocalisation qui les accompagnent: "The object of study is ultimately mobile and multiply situated, so any ethnography of such an object will have a comparative dimension that is integral to it, in the form of juxtapositions of phenomena that conventionally have appeared to be (or conceptually have been kept) "worlds apart" »(1998: 89). Il préconise donc une ethnographie multi-sites qui peut être réalisée de plusieurs manières ou grâce à différentes techniques : « [F]ollow the people, follow the thing, follow the metaphor, follow the plot, story or allegory, follow the life or biography, follow the conflict » (1998: 89), c'est-à-dire suivre les personnes, les choses, les métaphores, les complots, les histoires ou les allégories, les biographies ou les conflits. Appadurai est l'un des inspirateurs de cette multi-sited ethnography puisque, dans ses travaux, il n'a pas hésité à prendre la complexité des trajectoires individuelles et 
collectives des migrants et à pluraliser les lieux d'investigation afin de montrer le décalage entre l'implantation d'individus dans un site déterminé (le site) et leur "localité » entendue comme relation d'appartenance à un groupe dont les membres vivent dans un ou des pays différents (Abélès dans Appadurai 2001: 21).

En m'inspirant de ces réflexions, outre le premier niveau de comparaison établi entre les représentations de Bretons ayant maintenu et construit des liens avec leur culture et leur territoire d'origine et ceux ayant rompu ces liens, j'ai également souhaité établir un deuxième niveau de comparaison en «suivant les gens » (follow the people), c'està-dire en rencontrant les Bretons retournés vivre dans leur territoire d'origine après une migration plus ou moins longue au Québec. Marcus souligne que cette technique est la plus conventionnelle en ethnographie multisites et que les migrations sont un des objets de recherche contemporains des plus ordinaires : " this technique is perhaps the most obvious and conventional mode of materializing a multi-sited ethnography. [...] Migration studies are perhaps the most common contemporary research genre of this basic mode of multi-sited ethnography » (1998: 90). Ainsi, j'ai comparé les propos que tiennent mes interlocuteurs sur leur appartenance culturelle avant et après leur migration au Québec, ainsi que de ceux retournés vivre dans leur territoire d'origine. Green qualifie ce type de comparaison de «modèle linéaire » : "Suivre le migrant sur son chemin de Vilno à New York, de la Vénétie à Paris, d'Algérie en France, le comparer en quelque sorte avant et après, implique une comparaison du pays d'origine avec le pays d'arrivée »(Green 2002 : 27). À mon sens, il s'agit ici d'établir davantage une appréhension continue qu'une comparaison en tant que telle de parcours migratoires et de représentations. Pour cela, je suis retournée en Bretagne à l'hiver 2007. J'ai cherché à retracer le parcours de migration de mes interlocuteurs, les conditions de leur retour en terre d'origine et les représentations de leur appartenance culturelle dans chacun des territoires. En suivant les conseils de Marcus, j'ai pu ainsi établir des connexions entre les différentes bribes de discours recueillies dans chaque site : «[T]he persuasiveness of the broader field that any such ethnography maps and constructs is in its capacity to make connections through translations and tracings among distinctive discourses from site to site» (1998: 84). Selon lui, ce mode de construction de la recherche multi-site est tout indiqué pour lier les lieux de production de culture entre lesquels il n'y avait pas 
nécessairement de liens évidents : "This mode of constructing multisited research is thus especially potent for suturing locations of cultural production that had not been obviously connected» (1998: 93). Dans l'un de ses articles, Guilbert insiste sur l'intérêt de cette méthode pour l'ethnologue : «En étudiant le fait migratoire, au lieu de se fixer un lieu d'observation fixe, l'observation in situ, l'ethnologue va davantage suivre le mouvement des individus et des groupes dans leurs pérégrinations. [...] L'ethnologue se fait lui-même migrant » (2005: 8).

Marcus précise cependant que la méthode comparative n'est plus empruntée de nos jours uniquement pour mettre en parallèle deux sites physiques, mais aussi pour interroger différents niveaux de la population migrante (hommes, femmes, adultes, jeunes, pionniers, nouveaux migrants, etc.) (1998). C'est la démarche que j'ai adoptée en analysant les propos de personnes ayant migré dans la période couvrant les années $1950^{5}$ à 2005, afin de rendre compte, avant la disparition des anciennes générations, de l'évolution des conditions de migration. Ce type de comparaison m'a permis de mettre en lumière, dans l'une des parties de ma thèse, les transformations qui se sont opérées entre les anciennes et les nouvelles migrations : les motivations des migrants, la catégorie socioprofessionnelle dont ils sont issus, les emplois occupés dans le pays d'accueil, les liens tissés dans le territoire d'accueil et ceux construits ou maintenus avec le territoire d'origine.

Maîtrisant la langue bretonne, j'ai souhaité également apporter une recherche originale en dévoilant les représentations de quelques migrants bretonnants (ceux qui parlent le breton) dans leur propre langue (acquise par transmission familiale ou apprentissage) ${ }^{6}$. Les sentiments et représentations sont parfois plus faciles à exprimer dans sa langue maternelle ou «la langue du cœur». De plus, je pense comme l'affirme Marcus que cette connaissance de la langue du groupe de référence étudié est importante dans l'étude multi-sites et permet même une plus grande exactitude dans la compréhension et l'analyse. Pour le

5. «Au total, on peut dire que la Bretagne a fourni au Canada deux vagues de pionniers. Les premiers sont arrivés aux XVII et XVIII ${ }^{\mathrm{e}}$ siècles et ils se sont irrémédiablement fondus à la collectivité. La seconde vague de nouveaux arrivants est débarquée à partir des années 1950 et forme une communauté canado-bretonne plus consciente de ses traits culturels propres » (Mimeault dans Magocsi 1999 : 282).

6. En m’appuyant sur les travaux réalisés par Elli Kaïja Köngas-Maranda (dans Mathieu 1983). 
paraphraser, elle garantit l'intégrité d'un terrain traditionnel et donne au champ délimité - une personne, un groupe de référence ou une communauté - sa plus grande cohérence en tant que culture (1998: 92).

En résumé, dans une approche comparative, j'ai choisi volontairement d'interroger trois catégories de migrants bretons : des migrants revendiquant et valorisant leur appartenance et leur différence culturelle bretonne plutôt que française en terre d'accueil québécoise, bien qu'éloignés de leur territoire d'origine; des migrants, résidant au Québec, se considérant " plus Français que Bretons », voire ayant rompu les liens avec leur culture et territoire d'origine et ne cherchant pas à se rapprocher de la communauté bretonne du Québec; des migrants du Québec retournés vivre en Bretagne, dénommés «migrants de retour ». Au total, trente-cinq personnes ont été interrogées.

\section{Taille du corpus et justification}

Pour trouver des Bretons ayant maintenu et construit des liens avec leur culture et leur territoire d'origine, j'ai utilisé, d'une part, la liste des membres de la seule corporation de Bretons au Canada, l'Union des Bretons du Canada, à Montréal, après autorisation du président de cette association. D'autre part, ayant établi de nombreux contacts préalables en Bretagne avec les associations de familles de migrants, ainsi qu'avec des migrants de retour en Bretagne, j'ai construit un réseau informel me permettant de trouver des interlocuteurs potentiels au Québec par le biais d'autres migrants. Par ailleurs, mes contacts, mes opportunités de terrain et ma double appartenance «française » et «bretonne migrante », du moins momentanément, m'ont permis de rencontrer des Bretons ayant rompu les liens avec leur culture et leur territoire d'origine. J'ai donc adopté trois types de méthodes d'échantillonnage, l'une que l'on pourrait qualifier de « recherchée » (informateurs rencontrés par recherche dans des réseaux communautaires); l'autre, de «boule de neige " (informateurs rencontrés par le biais d'autres informateurs) et la dernière enfin $d^{\prime}$ ' accidentelle ».

En suivant les conseils de Monsutti (2005 : 48), j’ai favorisé l'exemplarité des personnes choisies plutôt que leur représentativité quantitative. J'ai donc réalisé trente-cinq récits de vie et entretiens auprès de mes interlocuteurs : vingt-deux migrants de première génération résidant au Québec et treize migrants de première génération 
de retour dans leur territoire d'origine ${ }^{7}$. Ainsi, cet échantillon m'a offert une variété d'expériences suffisante pour atteindre ces points de régularité et de saturation dont parle Monsutti et ainsi me permettre d'identifier clairement des représentations et pratiques communes ou divergentes entre les migrants bretons rencontrés et selon les contextes.

[La] représentativité des exemples choisis [est garantie] par la profondeur des données accumulées: en recueillant des histoires de vie circonstanciées, on découvre une foule de liens reliant de nombreuses personnes; en recoupant les trajectoires individuelles, on obtient un témoignage représentatif et vivant. La pertinence et la fiabilité des données est alors établie par la régularité (lorsqu'une information est fournie indépendamment par plusieurs personnes) et par la saturation (lorsque aucune donnée nouvelle ne peut être obtenue par des entretiens ou des observations supplémentaires) (Monsutti 2005 : 48).

Ma recherche est donc exploratoire et vise l'exemplarité, non l'exhaustivité. La taille du corpus m'importe peu. Ce qui m'intéresse, c'est la représentativité des discours, leur répétition, leurs contradictions, leur complémentarité pour une meilleure compréhension de la construction identitaire de mes interlocuteurs.

Géographiquement, j'ai donc limité mon terrain de recherche à la province de Québec et à la région Bretagne ${ }^{8}$. Mais pourquoi ne pas avoir choisi d'étendre cette recherche à l'ensemble du Canada? Outre la faisabilité du projet, j'ai rapidement trouvé plus cohérent de comparer deux espaces ayant des points communs en termes d'échelon territorial (province/région) et d'histoire culturelle (revendication linguistique). À titre indicatif, voici le lieu de provenance en Bretagne des trentecinq migrants bretons interrogés: Finistère (16), Morbihan (10), Côtes d'Armor (6) et Ille-et-Vilaine (3). Concernant la répartition géographique dans le territoire d'accueil, même si la plupart se trouvent

7. Auxquels s'ajoutent treize migrants de seconde génération dont quatre sont retournés dans leur territoire d'origine, dont je n'ai pas eu le temps de retranscrire et d'analyser le discours, mais qui pourront faire l'objet d'une recherche ultérieure.

8. Afin de limiter mon terrain de recherche, j'ai préféré limiter le choix de mon corpus aux Bretons originaires de la Bretagne administrative actuelle, c'est-àdire constituée de quatre départements (Finistère, Morbihan, Ille-et-Vilaine, Côtes d'Armor). Cependant, il est important de préciser que nombre de Nantais, rattachés à la région Loire-Atlantique depuis la création des régions en 1982, se considèrent Bretons et le revendiquent. 
concentrés dans la ville de Montréal comme de nombreux autres migrants au Québec, j'ai mené une enquête de terrain tant en milieu urbain, qu'en milieu rural, dans les grandes villes comme Montréal (22) et Québec (6) et dans des endroits très isolés en région (7), comme en Gaspésie (à l'est de Québec) ou dans les Laurentides (au nord de Québec). En effet, comme le souligne Fardon, « tout autant que l'analyse des espaces centraux, l'observation des espaces jugés périphériques et des dynamismes locaux permet d'appréhender les tensions et les enjeux du monde moderne» (Fardon 1995 : 4). Autrement dit, tout autant que les lieux centraux de concentration des migrants, tels qu'à Montréal et Québec, les régions où les migrants sont plus isolés peuvent permettre d'observer des processus de construction identitaire particuliers. En Bretagne, «le territoire du retour », les treize personnes interrogées se répartissent ainsi dans les quatre départements : Finistère (9), Ille-etVilaine (3) et Morbihan (2).

Concernant les périodes de migration de mes interlocuteurs, le corpus choisi se répartit de manière assez homogène entre 1949 et 2005, avec une plus forte représentation pour les périodes de l'après-guerre (les années 1950-1960) et des années 1980-1990.

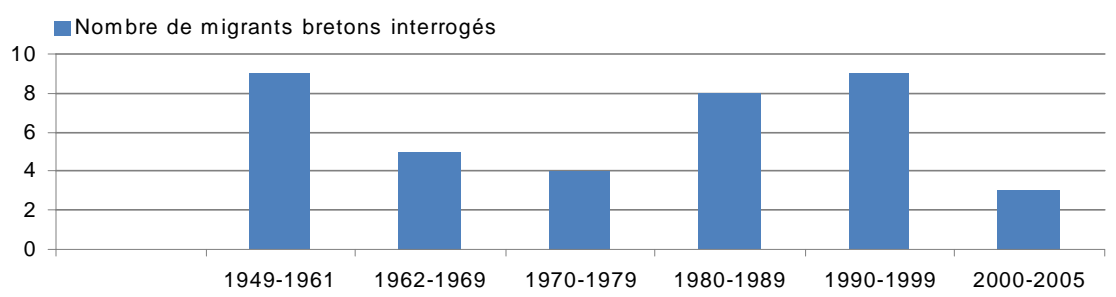

Fig. 1 : Répartition des Bretons interrogés selon leur période de migration.

Cependant, ma recherche, de type qualitative, est davantage centrée sur le contenu du discours de mes interlocuteurs à propos de leurs trajectoires migratoires que de leur représentativité quantitative. Je me base donc davantage sur les répétitions, les contradictions, les nuances dans les propos de mes interlocuteurs.

Deux autres indicateurs peuvent être intéressants pour l'analyse des trajectoires migratoires de mes interlocuteurs : l'âge et le sexe. En effet, l'âge auquel les migrants bretons sont arrivés en terre d'accueil peut être déterminant quant à leur capacité d'intégration et des liens maintenus avec leur territoire d'origine. Selon mon échantillon, les 
personnes ayant émigré entre les années 1949 et 1970 avaient entre dix-huit et vingt-cinq ans (seules deux personnes avaient trente-cinq et quarante ans, car issues d'une première migration vers Paris); tandis qu'après les années 1980, l'âge moyen de migration se situe entre vingtcinq et trente-cinq ans. Après les années 2000, la tranche d'âge des migrants est supérieure à trente ans (fig.2). Chronologiquement, les Bretons semblent donc émigrer de plus en plus tard au cours de leur vie. Ce constat peut être certainement mis en corrélation avec la plus grande accessibilité aux études au cours du temps et par conséquent un départ à l'étranger de plus en plus tardif en vue d'une insertion professionnelle. Selon les témoignages recueillis, la plupart des Bretons rencontrés sont partis vers «le Nouveau-Monde ${ }^{9}$ " soit en tant que jeune célibataire, jeune couple ou parfois avec un ou deux enfants en bas âge. Mais cela est valable pour la plupart des migrants du Québec puisque les politiques de migration canadienne et québécoise ont toujours privilégié l'accueil de jeunes migrants afin de mettre un frein au vieillissement de la population, à la pénurie de main-d'œuvre et à la dénatalité. De plus, depuis 1968, le gouvernement fédéral possède un Service de l'immigration distinct et cherche à renforcer le poids des francophones dans un Canada majoritairement anglophone. Les jeunes diplômés francophones avec des enfants en bas âge cumulent donc toutes les chances d'être acceptés sur le territoire.

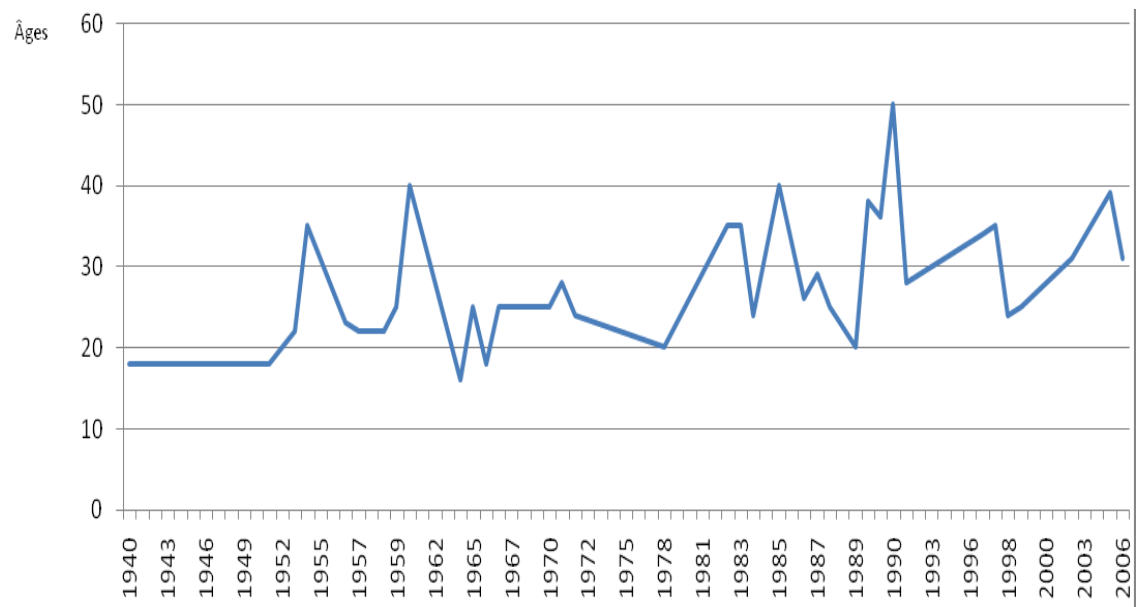

Fig. 2 : Âge des migrants bretons interrogés au moment de leur départ de Bretagne pour le Québec

9. L'utilisation répétitive de ce terme par mes interlocuteurs pour désigner leur 
Concernant le sexe de mes interlocuteurs, un quart seulement d'entre eux sont des femmes. De ce point de vue, mon corpus semble représentatif de la présence française au Québec puisque le recensement général de 2001 de la population française migrante au Québec comptabilise également davantage d'hommes $(52,6 \%)$ que de femmes $(47,4 \%)$ (Ministère de l'immigration et des communautés culturelles 2005). Cette tendance rappelle les données établies par Le Clec'h au siècle dernier. En effet, si je me réfère aux analyses menées par cet historien breton, contrairement au processus de migration interne des Bretons vers Paris ${ }^{10}$, le pourcentage d'hommes partis seuls, plus précisément non mariés, au Canada a été plus important que celui des femmes à la fin du XIX et au début $\mathrm{XX}^{\mathrm{e}}$ siècle. Comme le souligne Green, cet état de fait valable pour la plupart des populations migrantes expliquerait la tendance des études migratoires à considérer pendant longtemps le migrant comme une figure masculine, un aventurier ou un travailleur migrant. Par conséquent, jusqu'à une période récente, les femmes ont été largement absentes des discours et des imaginaires concernant les mouvements de population. Cependant, en ce début de $\mathrm{XXI}^{\mathrm{e}}$ siècle, "il semble plus que nécessaire d'intégrer les femmes dans une réflexion sur les migrations ", recommande Green (2002 : 34). Il ne s'agit pas ici de faire une analyse de la femme bretonne migrante, mais de voir comment ses représentations de son appartenance culturelle en contexte de migration peuvent questionner autant que celles de l'homme breton migrant, pour en relever les différences.

\section{Le lieu et la fréquence des entrevues}

La plupart de mes informateurs m'ont reçue, selon leur choix, à leur domicile, dans la cuisine ou la salle à manger, excepté quelques propriétaires de crêperies ou d'entreprises que j'ai rencontrés sur leur lieu de travail. Il m'a été d'ailleurs plus facile de capter les émotions de mes interlocuteurs à domicile, dans leur espace privé. En suivant les méthodes de Dubois-Ouellet (1994), je les ai revus à plusieurs reprises, sur de courtes séances, afin d'avoir une collecte de meilleure qualité. Ainsi, dans l'intervalle, j'ai eu le temps de réécouter l'entrevue et de repérer les points à approfondir. L'interlocuteur a eu, lui aussi, le temps de rassembler ses souvenirs et de rechercher des documents importants

destination rappelle bien sûr les premières explorations du continent américain, telles que réalisées par Jacques Cartier.

10. Dans lequel les femmes étaient majoritaires. 
pour nourrir son témoignage. Cette méthode s'est révélée assez longue, mais comme le souligne Monsutti, «seules des rencontres répétées, dans l'espace et dans le temps, permettent de construire des relations de qualité qui sont une source privilégiée à la fois d'informations et d'émotions» (2005: 49).

\section{L'interaction entre moi " chercheur " et mes interlocuteurs : une démarche réflexive}

En m'inspirant des réflexions de Ghasarian (2002), au-delà du seul statut d'informateur, j'ai considéré mes interlocuteurs comme de réels acteurs participant à ma recherche, dans un souci de réflexivité. Le choix de cette méthode de réflexivité chercheur/ sujets de recherche, additionné à des rencontres régulières avec mes interlocuteurs lors de plusieurs activités m'ont permis d'établir un climat de confiance et d'améliorer la qualité de ma recherche.

De plus, j'ai utilisé avec profit ma position de Bretonne et/ou ma position de Française, dans tous les cas, migrante au Québec, pour rentrer facilement en contact avec mes interlocuteurs. Tel que le souligne Marcus, j'ai adopté la démarche d'un ethnographer-activist en renégociant mes identités en fonction de la situation où je me trouvais afin d'en apprendre davantage sur mes interlocuteurs (1998: 98). Par exemple, auprès des Bretons attachés à leur appartenance culturelle régionale, je me présentais en tant que bretonne, faisant partie de la même «tribu », « des leurs », de surcroît migrante. Ainsi, je n'ai pas eu beaucoup de difficultés à "briser la glace». Je me suis servie de ce statut pour comprendre «du dedans» leurs expériences migratoires. J'ai adopté un « regard rapproché », propre à l'ethnologie du proche ${ }^{11}$.

À ce sujet, Monsutti souligne que «(1)a relation entre l'ethnologue et son informateur est [...] ambiguë. Le premier est payé pour entrer en contact, il vient de loin, il recueille des informations et repart [...] Pour lui, s'investir personnellement dans les relations est le seul moyen de recueillir des données fiables [...] La limite entre l'utilisation légitime de ces contacts et l'indécence est ténue » (2005: 49). Les lectures de Ghasarian m'ont éclairée sur les principaux conflits de ce type auxquels j'allais être confrontée sur mon terrain. Il écrit que «[1]e chercheur

11. Ce que l'on définit comme anthropology at home en Amérique du Nord a plusieurs dénominations en France : ethnologie ou anthropologie du proche, ethnologie à domicile ou encore ethnologie du monde contemporain. 
connaît une sorte de conflit existentiel entre le subjectivisme (la sensibilité) et l'objectivisme (la rigueur) d'une part, la bonne conscience due à l'idée d'utilité scientifique et la mauvaise conscience associée au fait d'être un témoin indiscret d'autre part»(2002: 24). Face à ce double conflit, Cohen conseille de conjuguer recherche et sensibilité. Cette association de l'objectif et du subjectif constitue ce que Wolcott (1995) appelle "l'art du terrain». Cet art est fondé sur un bricolage constant dans lequel la création d'un espace de confiance permet l'expression des émotions (Ghasarian 2002 : 26). Cependant, j'ai éprouvé parfois quelques difficultés à légitimer ma bretonnité auprès des Bretons du Québec de par mon accent du sud encore reconnaissable après dix ans de migration en Bretagne et le pluralisme de mes origines familiales. Comme me l'a fait remarquer un de mes interlocuteurs, « je ne suis pas une pur beurre!». Il existe en effet "des degrés de bretonnité » selon l'expression de P.J. Simon. Cependant, ma maîtrise de la langue bretonne, des danses et de la musique bretonnes m'ont permis de « justifier» mon appartenance culturelle bretonne. D'un autre côté, mon «métissage culturel » (Turgeon 2003) m'a ouvert des portes pour dialoguer avec ceux ayant tourné le dos à leur identité culturelle régionale. Paradoxalement, le défi de l'ethnologie du proche est aussi de conserver une certaine distance avec l'objet d'étude, mais une distance permettant de produire une connaissance du dedans (Ghasarian 2002 : 237). Il m’a fallu donc être vigilante à partager les émotions et les représentations de mes interlocuteurs tout en gardant une distanciation. Comme le décrit Ghasarian, le chercheur doit puiser dans ses ressources personnelles et opérer une gymnastique continue pour garder une distance intellectuelle sans être indifférent (2002: 25-26).

Dans un autre registre, Bertaux souligne que «l'une des conditions pour qu'un récit de vie se développe pleinement, c'est que le témoin soit saisi par le désir de se raconter et qu'il s'empare lui-même de la conduite de l'entretien» (1980: 209). C'est pourquoi j'ai veillé à ne pas interrompre mes interlocuteurs même si leurs propos s'éloignaient de temps à autre des éléments recherchés. D'autre part, j'ai également interrogé des couples, afin de faciliter la résurgence de souvenirs liés à l'expérience migratoire et au territoire d'origine. Concernant les migrants retournés vivre en Bretagne, j'ai été confrontée à certaines difficultés, notamment à celle de la non-valorisation de leur expérience migratoire : «Je n'ai rien d'exceptionnel à raconter, je n'ai pas eu une vie extraordinaire là-bas [...]». Il y a également des choses que mes 
interlocuteurs ont refusé de voir enregistrées, « une demi occultation du réel » selon l'expression de Dubois-Ouellet (1994). Il a été important de respecter leur choix et de le préciser par écrit par le biais d'un protocole d'entente ${ }^{12}$.

\section{Les récits de vie ou life history}

Le récit de vie désigne à la fois une méthode d'enquête et le produit de cette méthode, à savoir le témoignage recueilli. Cette approche biographique a donné lieu à de nombreux débats entre les tenants de la méthode quantitative qui se fondent notamment sur des statistiques et ceux de la méthode qualitative.

Sur mon terrain, les récits de vie m’ont permis de reconstruire le parcours migratoire de mes interlocuteurs, de préciser les éléments que je recherchais et ainsi de construire un solide guide d'entretien, donc d'entreprendre une démarche pleinement inductive. Simonne DuboisOuellet souligne que

[m]algré les difficultés rencontrées sur le terrain, c'est souvent la méthode idéale pour connaître non seulement la nature des faits mais aussi les perceptions que l'acteur social en a. [...] Ceux qui acceptent de confier leur récit de vie, les informateurs et les informatrices, racontent un événement ou expliquent une pratique, mais ils commentent également cet événement ou cette pratique. Ils relatent les faits avec les sensibilités dont ils étaient investis, hier ou aujourd'hui, ce qui leur donne sens et signification. [...] Il n'y a pas deux personnes qui vivent le même événement de la même façon (1994: 55-56).

En France, Bertaux a redonné au récit de vie toute sa pertinence comme outil d'enquête. Voulant préciser le vocabulaire, il a repris la distinction du sociologue nord-américain Denzin entre life history et life story (Denzin et Lincol 2003). Pour Dezin, life story, que Bertaux traduit par « récit de vie », désigne l'histoire d'une vie telle que la personne qui l'a vécue la raconte. Quant au terme life history, Dezin propose de le réserver «aux études de cas portant sur une personne donnée, et comprenant non seulement son propre récit de vie, mais aussi toutes sortes d'autres documents : par exemple, dossier médical, dossier judiciaire, tests psychologiques, témoignages des proches » (1980). Ma démarche a consisté à suivre cette seconde méthode, celle de la life

12. Soumis préalablement au Comité d'éthique de l'Université Laval. 
history, c'est-à-dire de recueillir et d'analyser les récits de vie, mais aussi les lettres de correspondance, les dossiers de migration, ainsi que les photographies de famille, les coupures de journaux, les prospectus, etc. Cependant, il m'a fallu développer une profonde connivence avec mes interlocuteurs pour qu'ils m'autorisent à exploiter ces documents. Pour cela, j'ai pris soin après chaque rencontre soit d'envoyer à mes interlocuteurs le texte retranscrit de leur entretien, soit de les solliciter à nouveau pour me confirmer certains détails, afin qu'ils restent maîtres de leurs témoignages.

S'il est possible d'établir une distinction entre le récit de vie (life story) et l'histoire de vie (life history), il existe aussi les récits de pratiques. Ceux-ci portent sur les pratiques professionnelles ou culturelles du témoin et des gens de son milieu (Dubois-Ouellet 1994). Sur mon terrain de recherche, j'ai recueilli les récits de pratiques à l'intérieur même des récits de vie. Tous mes interlocuteurs ont été amenés à me parler, entre autres, de leur pratique professionnelle liée à leur expérience de migration ; d'autres, de leurs pratiques culturelles, matérialisant leur appartenance culturelle bretonne (danses, musiques, chant, cuisine, langue bretonnes, etc.).

En utilisant cette technique de la life history, je me suis rapprochée d'une des techniques propres à la multi-sited ethnography: « the life history or biography », une forme de données ethnographiques particulièrement favorisée ces dernières années. Cependant, Marcus rappelle que cela n'a pas toujours été le cas puisque les récits de vie ont été jusqu'à très récemment peu utilisés : " the use of biographical narrative as a means of designing multi-sited research rarely has been considered» (1998: 94). Fischer est l'un des rares à avoir produit des réflexions sur l'utilisation des récits de vie et, d'après lui, « [life histories] are potential guides of the delineation of ethnographic spaces within systems shaped by categorical distinctions that may make these spaces otherwise invisible» (1991: 24-27). Les récits de vie permettent de mettre en lumière des données ethnographiques qui seraient restées dans l'ombre sans cette même technique. Selon Dubois-Ouellet (1994), le récit de vie et le récit de pratiques semblent être les meilleurs outils pour dégager les perceptions, les émotions, pour capter la quotidienneté de l'existence, laquelle est le support de l'événement. Le témoin ne se bornera pas à se raconter, il racontera aussi les autres dans la mesure où ceux-ci ont «fait partie» de sa propre existence; le récit pourra ainsi être mis en situation socioculturelle (Poirier et al. 1983 : 205, 353). En effet, le 
récit de chacun de mes interlocuteurs a éclairé les pratiques des autres membres de la communauté bretonne.

\section{Les entretiens semi directifs}

Dans un second temps, pour compléter les données recueillies grâce aux récits de vie, j'ai mené des entretiens semi directifs avec les mêmes interlocuteurs en vue d'identifier le rapport de mes interlocuteurs à leur culture et leur territoire d'origine. Pour cela, j'ai élaboré deux guides d'entretiens, d'une part à partir de mes premiers entretiens exploratoires et d'autre part à partir de mes variables de recherche construites sur des critères signifiants. Chaque guide d'entretien a été adapté à la population interrogée : l'un pour les migrants établis en territoire d'accueil, au Québec, l'autre pour les migrants retournés vivre dans leur territoire d'origine, en Bretagne.

Suivant la méthodologie d'analyse qualitative utilisée, j'ai interrogé les migrants bretons établis au Québec sur : leur état civil et leur milieu d'origine (renseignements généraux), les circonstances de leur migration, leurs modes d'ancrages territoriaux, leurs trajectoires professionnelles, leurs modes ou stratégies d'intégration au sein du territoire d'accueil, les liens gardés ou construits avec la Bretagne, les représentations de leur bretonnité, francité, québécité et canadiannité, les vecteurs de leur appartenance culturelle bretonne en terre d'accueil. Pour les migrants retournés vivre en Bretagne, se sont ajoutées bien sûr des questions portant sur les caractéristiques de leur retour, mais aussi sur les liens gardés avec le Québec depuis leur retour, ainsi que sur les représentations de leur bretonnité et francité depuis leur retour.

Les représentations que m'ont livrées les migrants rencontrés m'ont permis de dégager quatre cas de figure et de répondre à mes questionnements relatifs à leur appartenance culturelle.

\section{"Plus Breton que Français"}

Même s'ils sont perçus comme des Français par la plupart des Québécois, vingt-neuf des trente-cinq migrants interrogés ont déclaré se sentir exclusivement bretons et se présenter en tant que tels au Québec, comme l'affirme Christian (65 ans, émigré en 1968, de retour en 2003) : «Non, moi, je n'ai jamais dit que j'étais Français. Breton, toujours ». D'ailleurs, certains n'hésitent pas à afficher leur bretonni- 
té ${ }^{13}$ en terre d'accueil, tel Emmanuel (35 ans, émigré en 1998) qui proclame ainsi : «C'est pas parce que j'ai quitté la Bretagne pour venir au Québec que je ne suis pas Breton. Sur mon char [voiture], c'est marqué. Bientôt, je vais avoir un gwen ha du [drapeau breton] devant ma porte. Je suis Breton avant d'être Français!»

Emmanuel exprime encore le caractère transmis de son appartenance culturelle bretonne par le fait qu'on lui ait attribué le même prénom que son grand-père et que son deuxième prénom soit celui de Marie, la mère du Christ : "Je porte le prénom de mon grand-père et celui de la sainte Vierge qui protège notre famille. Toute la famille porte ce deuxième prénom ». Il est vrai que le prénom Mari, forme bretonne de Marie, est très répandu en Bretagne. Croix précise qu'avec celui de Jean c'est le prénom le plus courant en Bretagne ${ }^{14}$. En effet, « l'Église, au XIX ${ }^{\text {e }}$ siècle surtout, promeut très fortement le culte marial : $45 \%$ des Bretons portent alors Marie pour second prénom. Le choix du prénom est donc bien le reflet de fortes habitudes sociales et culturelles, renforcées par l'habitude, jusqu'à la fin du XIX ${ }^{\mathrm{e}}$ siècle, de donner le plus souvent à l'enfant le prénom de son parrain ou de sa marraine », explique-t-il (Croix 2001 : 803). Aujourd'hui, en Bretagne et plus encore à l'extérieur, le simple fait de porter le prénom de Mari ou Marie comme deuxième prénom, d'autant plus pour un homme, est perçu comme un signe fort d'appartenance culturelle. Mais d'autres prénoms sont aujourd'hui considérés comme marqueurs de bretonnité, tant en Bretagne qu'en migration. Nombre de mes interlocuteurs ont d'ailleurs choisi d'attribuer l'un de ces prénoms dits « bretons » à leurs enfants, pourtant nés au Québec, dans le souci de leur donner un ancrage culturel, une référence au territoire d'origine de leurs ancêtres. À ce sujet, Croix fait une observation intéressante.

Il est significatif de voir fleurir, dans la deuxième moitié du $\mathrm{XX}^{\mathrm{e}}$ siècle, de nombreuses formes bretonnisées de prénoms «français » (Annaïck, Loïc, Soazig, etc.) : ils expriment de manière techniquement artificielle, puisqu'ils n'ont aucun passé breton, mais

13. Par bretonnité, j'entends la manière de sentir faire partie d'une communauté, de vivre son identité bretonne, de «se dire breton », les représentations, valeurs et pratiques qui y sont liées.

14. Un échantillon de 6000 enfants nés au XIX ${ }^{\mathrm{e}}$ siècle livre ainsi 22\% de Jean, $37 \%$ de Marie, mais un seul Corentin et un seul Goulven, et seule la présence d'Yves au sixième rang des prénoms attribués aux hommes marque une certaine originalité. 
sincère un attachement au patrimoine breton, servi par la publication, depuis la fin des années 1970, de nombreux petits catalogues commentés de prénoms «bretons » et même «celtiques ». Il existe donc bien des prénoms voulus et perçus comme «bretons », et leur caractère statistiquement marginal renforce encore cette perception (2001: 803).

Pour les migrants rencontrés au Québec, donner à leurs enfants un prénom dit «breton » témoigne en effet de leur attachement à leur appartenance culturelle régionale et de leur désir de transmission de cette particularité.

Certains migrants parlent surtout de leur lieu de naissance et d'enfance et de la transmission de la langue bretonne comme symbole de leur bretonnité. Par exemple, Henri (67 ans, émigré en 1952 à l'âge de 12 ans avec ses parents) explique que malgré ses cinquante-cinq années de migration au Québec, le lieu de sa naissance et de son enfance restera toujours le lieu de son appartenance culturelle : «Memep graet hon neizh amañ met, evel-just omp stag deus al lec'h ma'z omp ganet. [Même si on a fait notre nid ici, on restera toujours attaché au lieu où l'on est né] ». D'ailleurs, celui-ci n'hésite pas à afficher son appartenance culturelle bretonne en s'investissant depuis son arrivée au Québec au sein de l'Union des Bretons du Canada, mais aussi en pratiquant et transmettant sa langue maternelle bretonne. Roland (émigré en 1954), célèbre cinéaste au Québec, affirme encore avoir toujours gardé l'appartenance culturelle régionale qui lui a été transmise par ses parents : «Mon tout jeune âge m'a beaucoup influencé en Bretagne. Le fait que mes parents parlaient breton [...] Depuis, j'ai toujours été intéressé par la Bretagne. Tout le temps. J'ai toujours gardé un lien avec la Bretagne. Par exemple, je ne pourrais pas vivre à Paris. D'ailleurs quand je vais en Bretagne, je descends à Nantes. Je ne descends pas à Paris. Ça ne m'intéresse pas du tout. J'y ai passé trois, quatre ans, pour moi c'est suffisant. J'aime mieux être en Bretagne, voir un tas de gens qui m'intéressent. Je n'ai jamais pu me détacher de mon appartenance à la Bretagne. Ça oui. Je vous l'avoue franchement».

Pour certains migrants, l'expression de leur bretonnité semble d'ailleurs avoir d'autant plus d'importance qu'ils sont éloignés physiquement de leur territoire d'origine. Par exemple, Ifig (34 ans, émigré en 2000) affirme: «Je me sens foncièrement breton, encore plus ici. Ma culture bretonne, évidemment ça a plus de signification ici ». Youna (64 ans, émigrée en 1965, de retour en 2000) explique 
encore que certaines barrières ou certains conflits qu'elle ressentait entre communes en Bretagne se sont effacés au Québec où les migrants se rattachent à une même origine: "Je me suis toujours sentie très vannetaise en Bretagne, mais surtout bretonne là-bas [au Québec]. Il n'y avait pas de querelles de clochers ». En effet, dans la société rurale traditionnelle, une certaine rivalité existait entre paroisses bretonnes. Ces rivalités se manifestaient dans certains domaines : musique, danses, travail. Dans la société bretonne contemporaine, ces rivalités ne sont plus aussi marquées, mais elles restent tout de même présentes dans l'imaginaire collectif et sont entretenues par le biais des nombreux concours de danses, sonneurs, bagadoù, chanteurs de kan ha diskan, etc. En terre de migration, ces rivalités ne semblent plus avoir d'importance et une certaine solidarité existe entre Bretons.

Parallèlement à cette affirmation culturelle bretonne, la plupart de ces migrants ont une perception négative de ce que la France, leur nation administrative, leur propose pour valoriser cette bretonnité. Par exemple, Roland (86 ans, émigré en 1954) affirme : "les Français pensent que la France entière est unie, que tout le monde parle pareil, que tout le monde a la même origine; et s'ils savent qu'on est de différentes origines, ils ne veulent pas en entendre parler. C'est pour ça que le gouvernement de Pétain a retiré la Loire-Atlantique de la Bretagne. Pour eux, ce n'était rien, ce n'était pas important. Si la LoireAtlantique pouvait revenir en Bretagne, ça serait formidable, ça ! Là, il faudrait faire un film!» De même, Denis (39 ans, émigré en 2005) explique : "Moi, je me sens une appartenance à la Bretagne, mais la Bretagne pour moi n'est pas le paradis sur terre, sinon j'y serais resté. Par contre, si un jour on me propose un passeport breton, alors là je signe de suite. Aujourd'hui, je ne vois pas à quoi ça peut servir de se dire breton puisqu'il n'y a pas de reconnaissance ». Le témoignage de ces migrants traduit un certain rejet du système administratif français, ainsi que de certains comportements de leurs compatriotes. Ce sentiment a sûrement été accentué par le fait que ces migrants ont connu d'autres modes d'organisation et manières de vivre au Québec. En ce sens, leur expérience migratoire a fortement conditionné leur détachement de cette francité.

En réalité, ces migrants ont exprimé ouvertement avoir abandonné leur appartenance culturelle française. La seule francité qu'ils ressentent est celle attribuée par le regard de "l'Autre », c'est-à-dire certains Québécois qui les perçoivent comme des Français, voire des «maudits 
Français »: «Je ne suis Français que pour les Québécois », dixit Denis (39 ans, émigré en 2005) ; «Moi, je ne me sens pas du tout Française. Je n'ai plus aucun papier français. Je ne vote plus en France. Ça ne m’intéresse pas. Même si pour les Québécois, je reste une Française, une minorité audible », dixit Caroline (47 ans, émigrée en 1984) ; «Je me sens encore très Breton, mais je ne me sens plus Français depuis très longtemps ", dixit Philippe (49 ans, émigré en 1984). En terre d'accueil, ces migrants ont donc clairement tourné le dos à leur nation administrative, mais pas à leur «nation de cœur », c'est-à-dire la Bretagne. D'ailleurs, mes interlocuteurs n'ont pas fait de demande d'immatriculation au Consulat français, ce qui témoigne de leur désintérêt à être reconnu comme migrant français au Québec. Si certains ont entrepris la démarche, c'est simplement pour des questions administratives, tel Ifig (35 ans, émigré en 2000), immatriculé au Consulat français deux ans après sa migration, pour son mariage avec une québécoise : «Je me suis immatriculé au Consulat français parce que j'avais mon passeport à refaire puis le livret de famille quand je me suis marié ", explique-t-il. Le désengagement national de ces migrants est le reflet de la non-représentativité de la nation française, de l'affaiblissement de son autorité et de la perte de son influence auprès de certains citoyens français migrants. En se référant aux réflexions de Tajfel, on peut dire que si ces migrants bretons ont abandonné leur appartenance culturelle française, c'est parce que la France ne satisfait plus leurs besoins de construire leur identité sociale, notamment en ne souhaitant pas reconnaître leur spécificité bretonne qu'ils ont choisie ou acquise : "If a group does not satisfy this requirement, the individual will tend to leave it unless : (i) leaving the group is impossible for some "objective" reasons, or, (ii) it conflicts with important values which are themselves a part of his acceptable self image » (Tajfel 1978: 62). Si ces migrants bretons rejettent leur francité, ce n'est pas parce qu'ils ne se sentent pas réellement Français, mais plutôt parce que la France n'accepte pas de reconnaître leur bretonnité et ne leur permet pas de la vivre pleinement ${ }^{15}$. En ce sens, comme le postulent Shiose et Zylberberg

15. Notamment par les actes commis dans le passé pour dévaloriser la langue bretonne et par la non-reconnaissance des langues minoritaires en France, dont le breton. En effet, depuis la Révolution jusqu'à aujourd'hui, l'État français a cherché à éradiquer les langues régionales sur l'ensemble de son territoire, la République ayant choisi comme langue unique le français. Broudig détaille cette tentative d'éradication des langues régionales : «[Elle] prend surtout place sous la III ${ }^{\text {e }}$ République lorsque l'instruction publique est rendue 
en s'inspirant de Weber, « le pouvoir politique joue un rôle fondamental dans la formation, la structuration et l'organisation de l'identité culturelle» (Shiose et Zylberberg 1998: 108-109). Ces migrants se trouvent donc dans une forme d'appartenance en tension puisqu'ils dissocient, voire opposent, l'appartenance culturelle qu'ils ont choisie et dans laquelle ils se reconnaissent, leur bretonnité, de celle que le pouvoir politique leur a administrée, leur francité.

Concernant leur bretonnité, j'ai constaté que, d'après leurs discours, la plupart des migrants rencontrés sont fiers de leurs origines bretonnes, du territoire où ils sont nés, de leurs racines familiales et la migration ne saurait les altérer. Bien au contraire, pour certains, c'est justement leur expérience migratoire qui leur a révélé l'importance de cette bretonnité qu'ils ont découverte ou redécouverte en terre d'accueil.

\section{La découverte ou redécouverte de la bretonnité}

En effet, pour certains migrants, j'ai pu relever que ce choix d'appartenance culturelle à la Bretagne ne s'est pas édifié de manière linéaire, mais s'est façonné, s'est transformé, a fluctué avec le temps, suivant l'âge du migrant et en fonction de ses expériences en terre d'origine et d'accueil, en particulier pour les plus anciens d'entre eux.

Certains ont dans un premier temps rejeté, en migrant, leur appartenance culturelle bretonne, opérant ainsi une réelle migration de rupture comme la définit Rosental (1990: 1407-1410). C'est le cas par exemple de Zina (56 ans, émigrée en 1975) qui explique avoir rejeté sa famille, mais aussi "une Bretagne dure et triste ». Elle a également rejeté inconsciemment une Bretagne qui lui refusait d'apprendre et d'exprimer sa langue maternelle puisque sa mère n'a pas souhaité lui transmettre le breton. Son appartenance linguistique lui a

obligatoire. Les pouvoirs publics désirent assurer l'unité française et faciliter la promotion sociale au sein de la Nation. Pour ces raisons, les responsables de l'enseignement public commencent à proscrire l'usage de tous les patois ou parlers régionaux à l'école. À partir de la fin du XIX ${ }^{\mathrm{e}}$ siècle, les langues régionales sont interdites dans l'enseignement, au grand dam d'une partie du corps enseignant qui se demande comment enseigner dans de telles conditions à des élèves non francophones. Ainsi en 1902, le ministère Combes promulgue par décret l'interdiction de l'usage abusif du breton. Les écoles religieuses suivent rapidement et le breton n'est plus enseigné à partir du début du $\mathrm{XX}^{\mathrm{e}}$ siècle mais continue à être transmis de génération en génération par voie orale » (Broudig $1995)$. 
donc été interdite, comme elle l'exprime : "Mais c'est ma mère qui n'aurait pas voulu nous parler breton. Pour ma mère et pour mon père aussi sûrement, le fait qu'on soit élevés en français était une valeur positive parce qu'eux, surtout ma mère, avaient tellement souffert. Quand elle est allée à l'école, elle ne parlait pas français. Le breton était une langue que l'on entendait, sauf que ce n'était pas une langue dans laquelle on s'adressait aux enfants. C'était une langue qui était condamnée d'emblée, puisqu'on ne pouvait pas parler en breton. Je sais qu'une fois j'avais répondu en breton à ma grand-mère. Ça avait fait un effet! Comme si j'avais fait quelque chose qu'il ne fallait pas, mais surtout quelque chose qui était d'un comique extraordinaire. Ça ne paraissait pas naturel dans ma bouche. C'est quand même assez choquant que ce qui serait ma vraie langue maternelle produise un effet comique. Mais ça a été dur. J'avais essayé de parler et ça n'avait pas été pris au sérieux ». En effet, la plupart des Bretons de la génération de Zina ou des générations précédentes ont connu les conséquences de la dévalorisation de la langue maternelle bretonne par l'État français qui, à partir du milieu du XIX ${ }^{\mathrm{e}}$ siècle, réprimait les langues dites régionales et les présentait comme arriérées, rétrogrades et freins au progrès. L'usage de la langue bretonne dans la sphère privée n'était certes pas interdit par l'État, mais sa dévalorisation d'un point de vue social et économique a découragé les familles bretonnantes à transmettre leur langue maternelle.

Dans un premier temps, Zina a ainsi tourné le dos à son espace d'origine qui ne voulait pas lui transmettre sa langue maternelle et a envisagé tous ses projets dans le cadre de son territoire d'accueil, le Québec. La perception négative qu'elle avait de son groupe de référence d'origine, c'est-à-dire breton, a donc structuré son choix d'appartenance culturelle, comme l'explicite Tajfel (1978: 62). Paradoxalement, son expérience migratoire lui a aussi permis de prendre conscience de la valeur positive de son groupe d'origine, ce qui l'amenée à se rapprocher de son appartenance culturelle bretonne, de nombreuses années après avoir quitté la Bretagne : «Oh! Sur trente ans, j’ai beaucoup changé, mais au départ, j'étais volontairement loin de la Bretagne. Mais depuis une quinzaine d'années, j'ai un grand intérêt pour la langue bretonne. À chaque fois que je peux, je m'y intéresse grâce à Internet, aux revues, aux livres, etc. J'ai prévu aussi d'aller en année sabbatique en Bretagne, à Rennes, pour éventuellement passer une maîtrise en breton. C'est un projet encore », explique-t-elle. Les réflexions de Maalouf m'ont été 
d'une aide précieuse pour mieux comprendre le comportement changeant de Zina au cours de sa vie envers son appartenance culturelle bretonne.

\begin{abstract}
On a souvent tendance à se reconnaître dans son appartenance la plus attaquée ; parfois, quand on ne se sent pas la force de la défendre [comme Zina durant son enfance], on la dissimule, alors elle reste au fond de soi-même, tapie dans l'ombre, attendant sa revanche; mais qu'on l'assume ou qu'on la cache, qu'on la proclame discrètement ou bien avec fracas, c'est à elle qu'on s'identifie (1998: 34).
\end{abstract}

La migration de Zina au Québec pendant trente-deux ans l'a donc conduite à déterrer son attachement à la culture bretonne et à son territoire d'origine qu'elle n'avait en réalité jamais enfouie complètement. Son expérience migratoire lui a permis de reprendre conscience de sa bretonnité, de la ressusciter en quelque sorte, comme elle l'exprime: "Cette migration, c'est un choix personnel parce que ce n'était pas obligé. Mais faire ce choix, ça m’a permis de comprendre le pourquoi et le comment de là-bas et ma bretonnité. Ceux qui restent sont peut-être moins attachés à ce sentiment d'appartenance. C'est comme une langue, quand on n'en parle pas une autre, on ne reconnaît pas la richesse de la nôtre ». La perception de «l'engagé » s'oppose ici à celle de "l'attaché », selon la théorie de Sencébé. Pour la figure de «l'engagé », l'expérience d'un ailleurs révèle la valeur de ce qu'il a vécu dans son territoire d'origine (2004: 24-25). Mais le cas de Zina n'est pas isolé. Ainsi, Angèle (58 ans, émigrée en 1978) a davantage pris conscience de la beauté de son territoire d'origine et de son attachement à la Bretagne en migrant, ainsi qu'elle l'exprime : «Brav e oa memestra e Breizh! Met pa vevez aze just en Breizh, ne welez ket se. Nann, ret eo mont kuit ha aze lârez "memestra 'm eus lezet un dra bennak brav dre amañ" [La Bretagne est belle quand même! Mais quand nous sommes là-bas, on ne le voit pas. Non, il faut partir pour se dire "On a laissé quelque chose de beau tout de même"] ». Youna (68 ans, émigrée en 1965, retournée en 2000) explique encore avoir connu ses premiers pas de gavotte, la plus populaire des danses bretonnes, en terre d'accueil, comme la plupart des Bretons ayant migré peu après la Seconde Guerre mondiale, car la danse bretonne n'était pas très populaire dans les années 1950-1960 en Bretagne : «En fait, c'est par le biais de cette association bretonne que j'ai réappris à danser les danses bretonnes que je dansais dans les kermesses jusqu'à mes seize ans environ. Alors que dans les années 50-60, quand nous sommes partis, ça avait 
complètement disparu en Bretagne. C'était le désert culturel à cette époque-là ». C'est donc en migration qu'elle a redécouvert les pratiques culturelles de son enfance. C'est aussi la migration qui lui a permis de renouer avec cet élément de sa culture bretonne, à son retour en terre d'origine : «D'ailleurs quand je suis revenue en Bretagne, j'ai recherché tout de suite des occasions de danser en fest-noz. Et au village, à Theix, c'est super. On fête le 14 juillet [fête nationale française], mais à la mode bretonne, le 13 juillet, avec de la musique bretonne. Alors là, je m'éclate! » En réalité, de nombreux Bretons ont découvert ou redécouvert leur bretonnité en quittant la Bretagne, comme Louis Bizien (décédé, émigré en 1954 à Montréal) qui avait déclaré à Le Clec’h avoir retrouvé son identité bretonne, comme beaucoup de migrants, au Québec (Gonidec 1979 : 5). Le même constat a été fait par Tardieu concernant les Bretons de Paris : «beaucoup de ceux qui sont nés en Bretagne se sont mis à la danse en région parisienne et ceci est d'autant plus vrai pour les natifs du pays gallo », précise-t-il (2003: 89).

Ce phénomène de redécouverte, en terre d'accueil, de l'attachement à leur territoire d'origine s'explique en partie par le fait que les Bretons des années 1950-1960, quittant une Bretagne assez pauvre, triste et honteuse, n'avaient pas une image très positive de leur région. Il n'y avait donc pas de fierté, bien au contraire, à se revendiquer Breton, tant en Bretagne, en France, qu'en terre d'accueil. De plus, le fait que l'État français ait déprécié tout ce qui touchait à un particularisme régional, dont particulièrement la langue bretonne, avait laissé des traces dans la mémoire collective. Mais petit à petit, leur terre de migration leur a permis de prendre conscience d'eux-mêmes et, comme le formule Lipiansky (1995: 35-40), de produire leur identité dans cet espace de transfert, dans ce lieu commun entre le moi et l'autre que représente le Québec.

\section{Une double, voire une triple appartenance}

Cependant, parmi mes interlocuteurs, deux personnes adjoignent tout de même une appartenance française à leur appartenance bretonne. Par exemple, Yvon (78 ans, émigré en 1951, de retour en 1954) affirme que sa bretonnité était moindre que celle d'autres migrants en terre d'accueil, car il se sentait aussi Français. Comme si l'une venait empêcher ou atténuer l'autre : «Au Québec, on était Breizh [Breton], mais moins que certains. Mais je me sentais français aussi ». Albert (80 ans, émigré 
en 1951, de retour en 2000), lui, affirme avoir vécu sa bretonnité et sa francité au Québec, à parts égales: «Mais mon identité bretonne, je ne l'ai jamais perdue parce que j'étais Français. Je suis toujours resté très français. J'écoutais TV5 tous les jours et j'étais toujours au fait de ce qui se passait en France ». Ces interlocuteurs possèdent donc une double appartenance, celle à leur culture ethnique, la Bretagne, et celle à leur culture française. Ces migrants, contrairement à ceux qui ont une image négative de la France, en grande partie parce que celle-ci s'oppose à une reconnaissance de leur spécificité bretonne, ont une image très positive de leur nation. Par exemple, Albert explique : «J'ai peut-être une certaine fierté de la France sans pour autant minimiser ou restreindre la valeur des autres cultures ou pays ».

Plus rares sont les migrants bretons se sentant «Québécois ». Seuls quelques-uns ont choisi cette appartenance culturelle, mais ne l'ont pas fait au détriment de leur appartenance bretonne et/ou française. En fait, ils ont adjoint à leur appartenance bretonne et/ou française, cette appartenance québécoise, par un processus cumulatif et non substitutif. Albert (80 ans, émigré en 1953), conjugue par exemple son appartenance originelle britto-française et son appartenance québécoise: «J'étais fier d'être Français et en même temps profondément Québécois. Pour moi le Québec était important dans mon cœur et il l'est encore d'ailleurs. J'intègre totalement ces deux identités et cela ne provoque aucun conflit ». Cette triple appartenance culturelle a d'ailleurs aidé Albert à ne pas avoir "le mal du pays ", comme il l'exprime: "Non, je ne peux pas dire que la France m'ait manqué, alors que je suis resté profondément Français. Cependant, comme je l'ai déjà dit, je suivais de très près ce qui s'y passait, gardant une fierté de mon pays, tant par son histoire, sa culture, ses paysages si variés mais, étant pleinement intégré au Québec, je n'éprouvais pas de manque. J'assumais mes deux nationalités. Après tout, les Québécois sont de même origine que nous. Notre histoire est la même ». Philippe (49 ans, émigré en 1984) explique encore que, pour lui, chacune de ses appartenances s'exprime dans des lieux précis : "Quand je joue en fest-noz, je me sens Breton, mais quand je rentre à la maison, je veux être Québécois ». Ronan (émigré en 1978), lui, bien que resté attaché à sa bretonnité, se considère aussi Québécois en terre d'accueil : "Moi je me suis toujours considéré comme un Québécois qui voulait partir [monter] une affaire au Québec, et non comme un migrant. On est 
Québécois, d'origine bretonne ${ }^{16}$. Zina (56 ans, émigrée en 1975) juxtapose quant à elle ses diverses appartenances. Elle se sent à la fois migrante dans la ville de Québec, bretonne dans son cœur, canadienne dans son territoire d'accueil et montréalaise à Montréal : « Je fonctionne comme n'ayant pas d'oppositions. Il n'y a pas d'oppositions entre la France, le Québec, la Bretagne. C'est en moi, ça fait partie de mon identité. Ça ne peut pas se définir d'un bord ou d'un autre ». Ces interlocuteurs ne conçoivent donc pas leur enracinement de façon médiocre, en un terroir qui ressemblerait à une réserve ethnographique, mais comme un enracinement dans le destin historique autonome d'une population, l'élargissant en cercles concentriques, plus ou moins nombreux, selon leur ressenti : une appartenance ethnique ${ }^{17}$ bretonne, une appartenance nationale française, une appartenance migrante québécoise, une appartenance fédérale canadienne, une appartenance citadine montréalaise, etc.

Les témoignages de ces migrants me rappellent les réflexions de Maalouf, écrivain libanais installé en France, sur la multi appartenance du migrant: "Ce qui fait que je suis moi-même et pas un autre, c'est que je suis ainsi à la lisière de deux pays, de deux ou trois langues, de plusieurs traditions culturelles. C'est précisément cela qui définit mon identité » (1998: 7). Certains migrants bretons du Québec semblent donc ressentir un profond métissage de leur identité en réclamant haut et fort l'appartenance à leur territoire de naissance (la Bretagne et/ou la France) tout en y ajoutant une identification à leur territoire d'accueil, le Canada, Québec ou Montréal, comme l'observent aussi Helly et al.

16. Témoignage donné dans le cadre d'une émission de TV5, « Bâtisseurs d'ailleurs », diffusée le 18 novembre 2006.

17. J'utilise ici le terme d'appartenance ethnique dans le sens d'appartenance, ressentie par les individus, à un groupe auquel leurs ancêtres, "réels ou symboliques », ont appartenu et/ou au devenir partagé d'un tel groupe, tel que défini par Isajiw (1999: 35). La définition du terme culture donnée par Texier rejoint d'ailleurs cette conception puisqu'il entend par culture, au sens ethnologique du terme, "l'expression profonde d'un vouloir vivre collectif. Elle donne à l'individu sa dimension sociale, non seulement dans l'espace, en tant que signe d'appartenance à une communauté déterminée, mais aussi dans le temps, dans la mesure où elle le relie à toutes les générations qui l'ont précédée, le faisant participer, sans qu'il s'en rende clairement compte, de la durée qu'elles représentent»(Texier 2004 : 22). C'est précisément sur cette acception de l'identité culturelle que porte ma recherche, en prenant comme cas d'étude celle des Bretons en situation de migration. 
chez les migrants marocains du Québec. Il n'y a donc pas dans ces caslà substitution d'une identité à une autre, mais davantage accumulation de plusieurs identités. Ces individus se comportent ainsi en parfaits migrants, s'adaptant à leur nouvel environnement (2001: 78). En paraphrasant Saint-Martin, on pourrait qualifier ces migrants bretons comme des « êtres de là » (Oury 1989 : 112) mentalement, et non simplement physiquement, autrement dit ce que Heidegger conceptualise par le dasein, c'est-à-dire " être là », " [n]on pas référence à l'espace mais, à la façon aristotélicienne, l'identification et l'appropriation d'une localisation » (Saint-Martin dans Jewsiewicki et Létourneau 1992 : 127). Cependant, d'après l'apport de Tajfel, ce choix de multiples appartenances culturelles n'est possible que dans la mesure où chacun des groupes auxquels l'individu décide de s'identifier lui apportent des éléments de satisfaction, des éléments positifs pour construire son identité : "It can be assumed that an individual will tend to remain a member of a group and seek membership of new groups if these groups have some contribution to make to the positive aspects of this social identity; i.e. to those aspects of it from which he derives some satisfaction» (1978: 62). Par exemple, Albert (80 ans, émigré en 1951, de retour en 2000), étant attaché à la fois à son appartenance culturelle bretonne, française et québécoise, a une image très positive à la fois de son territoire d'origine, de son territoire administratif et de son territoire d'adoption : « J'ai une image très positive de la Bretagne. C'est une région dynamique, avec beaucoup d'atouts. Elle exploite très bien son potentiel : agriculture, transformation des produits, mer (algues) et maintenant, importants projets en production électrique (hydroliennes). Ses villes et sa campagne sont très jolies et agréables. J'aime aussi le tempérament breton, son histoire ». Il ajoute : "J'ai une image assez positive de la France malgré certains comportements décrits précédemment. Les paysages sont jolis et variés, chaque région étant différente des autres. J'apprécie beaucoup la richesse de son patrimoine, de son histoire et de sa culture ».

Cependant, Maalouf avertit que ces formes d'appartenance multiples engendrent un rapport différent avec les autres comme avec sa propre «tribu » et peuvent entraîner des conflits ou des décalages culturels entre individus d'un même groupe, entre ceux « restés au pays » et ceux ayant migré. Il n'y a pas simplement «nous » et « eux », deux armées en ordre de bataille qui se préparent au prochain affrontement, à la prochaine revanche. "Il y a désormais, de "notre" côté, des 
personnes avec lesquelles je n'ai finalement que très peu de choses en commun [les Bretons demeurés au pays], et il y a, de "leur" côté, des personnes dont je peux me sentir extrêmement proche [les Québécois ou les autres migrants]», écrit-il (1998: 40).

Cependant, excepté ces deux cas de figure, rares sont les migrants bretons à exprimer une appartenance culturelle québécoise puisque beaucoup ont eu le sentiment et l'expérience d'être mal acceptés par leurs hôtes. Pourtant, certains aimeraient revendiquer pleinement leur double appartenance à la Bretagne et au Québec.

\section{Québécois de cœur, migrant de fait}

En effet, la plupart des migrants rencontrés m'ont confié se sentir très attachés au Québec et à sa culture francophone, comme l'exprime Henri (67 ans, émigré en 1952) : «Pa vezan amañ e vezan, ez on Kebekad. Hon bro evidomp eo ar c'hebek. N'eo ket an Ontario, ar Manitoba pe ar Stadoù-Unanet. Me zo stag deus ar C'hebek [Quand nous sommes ici, nous sommes Québécois. Notre pays, c'est le Québec. Ce n'est pas l'Ontario, le Manitoba ou les États-Unis] ».

Cependant, plusieurs considèrent que les mentalités de leur société d'accueil ne leur permettent pas d'assumer parfaitement leur double appartenance culturelle. Certains se sentent avant tout «migrants », c'est-à-dire comme des personnes attachées avant tout à un pays d'origine et peinant dans un contexte étranger à se créer une place sociale, comme le définissent Denise Helly et al. (2001 : 68). Par exemple, l'épouse d'Henri, Angèle (58 ans, émigrée en 1978) ne semble pas d'accord avec l'opinion de son mari et déplore être toujours considérée comme une étrangère dans la société québécoise. De colère, elle s'exprime sur ce sujet en partie dans sa langue maternelle, le français, et en partie en breton, la langue choisie par la famille: "Je suis Québécoise mais... Marteze en em santan e-giz une migrante [Peutêtre que je me sens comme une migrante]; alors je vais plus facilement vers les autres migrants. Et puis ils me rendent beaucoup plus aussi. De vrais amis québécois, on n'a pas réussi à s'en faire, pourtant on a essayé. C'est toujours superficiel. Quand on va plus loin, pfff! Alors qu'on a réussi à se faire de vrais amis italiens. Aesoc'h eo skoulmañ darempredoù gant tud deus broioù-all eget gant Kebekiz. Trist eo [C'est plus facile de lier des relations avec des personnes d'autres pays qu'avec des Québécois. C'est triste]. Moi j'ai déjà vu et entendu dire de mes enfants : Ce sont 
juste des migrants ! Ma bugale n'int ket evel se ! [Mes enfants ne le sont pas !]. Ils sont nés ici! Putain! [colère] Abalamour ma'z omp ganet en tu all on restera des déracinés, e vimp ordin etre an daou [Parce que l'on est né de l'autre côté, on restera toujours des déracinés, nous sommes entre les deux] ». Henri acquiesce, mais relativise la situation en misant sur le futur, ses enfants : «Ar pezh a lâr Angèle a zo gwir, evel-just eo start bezañ divroad. An divroidi eo start dezhe bevañ memes tra. Met pas evit ni bugale : a vevañ aman, zo ganet amañ, o deus graet o buhez amañ. Ret eo daou remziat evit dont da benn da goll-se [Ce que tu dis Angèle est vrai. C'est difficile évidemment d'être des migrants. Mais l'immigration est difficile à vivre pour les Québécois aussi. Cependant, pour nos enfants qui sont nés et qui ont fait leur vie ici, c'est différent. Il faudra deux générations pour que s'effacent ces tensions]».

Christian (émigré avec sa famille en 1968 à Rimouski, puis à Québec et à Montréal, de retour en 2003), apporte également un témoignage intéressant, allant plus loin encore puisqu'il considère qu'il n'y a pas d'intégration possible quel que soit le territoire d'accueil : «Je ne pense pas qu'une intégration existe vraiment. On s'acclimate aux gens ». Il souligne également le fait que sa migration l'a conduit à être perçu comme un migrant, non seulement dans son territoire d'accueil, mais aussi dans son territoire d'origine, à son retour de migration : "Quand j'étais enfant, on savait que j'étais breton et on me courait après dans la cour de l'école en me traitant de plouc ${ }^{18}$, comme si j'étais un étranger. Au Québec, bien sûr j'étais perçu comme un migrant. Et quand je suis rentré en France suite à mon expérience au Québec et ailleurs, j'étais encore perçu comme un migrant !». Ce sentiment de se sentir « étranger » où que l'on soit avait été également relevé par Sayad chez les Algériens en France dont il rapporte les témoignages.

Ici [en France], nous avons nos habitudes, que cela nous plaise ou non. C'est difficile de s'y arracher. Nous vivons dans ce pays depuis des années, mais ce n'est pas notre pays... Là-bas, c'est notre pays... Mais c'est un pays que nous portons dans notre cœur seulement. Nous faisons comme si nous ne l'avions pas quitté... À la fin, nous ne savons plus où nous sommes... (1986:45).

18. Le terme de ploucs désigne à l'origine les paysans et notamment les paysans bretons, en référence aux innombrables "plou » (qui signifie "paroisse » en breton) existant en Bretagne, comme par exemple Plouha, Ploumanac'h, Plouhinec, Plougastel. L'utilisation actuelle de ce terme revêt une forme péjorative. 
Finalement, le migrant finit par se sentir «étranger » où qu'il aille, qu'il demeure dans son territoire d'accueil ou qu'il retourne dans son territoire d'origine. La plupart des migrants bretons rencontrés ne se sentent pas appartenir à leur société d'accueil, canadienne ou québécoise et se sentent de moins en moins appartenir à leur société d'origine. Cette attitude ne tient pas à un refus de ces appartenances de leur part, mais davantage au sentiment de rejet qu'ils ressentent de la part de leur société d'accueil vis-à-vis de leur identité française et de leur éloignement progressif de leur société d'origine.

\section{Les citoyens du monde}

J'ai pu relever un autre cas de figure chez quatre de mes interlocuteurs : ceux qui ont une forme d'appartenance «labile», selon la définition de Sencébé, caractérisée par une extériorité dans leur rapport à l'espace (2004: 25). On est partout chez soi sans être de nulle part, se déclarant " citoyen du monde », comme Emmanuel (35 ans, émigré en 1998) : «En fait, je me sens de nulle part et de partout. Je ne me sens ni étranger en Bretagne, ni au Québec. Je me sens citoyen du monde. J'ai une identité bretonne parce que j'y tiens et que c'est ma culture, mais ça ne m'empêche pas de vivre ailleurs ». Un autre se sent à la fois francophone et citoyen du monde: "Francophone, surtout après ces trois années d'enseignement en français. La langue française est très importante pour moi. Mais je ne me sens pas Canadien et je pense que je ne me sentirai jamais Canadien. Moi je me sens très concerné par les problèmes environnementaux et je pense qu'il faut se sentir citoyen du monde, penser globalement », dixit Drian (34 ans, émigré en 1997). Par contre, il rejette en partie sa bretonnité ou plus précisément sa gallésité ${ }^{19}$ car l'image négative qu'on lui a transmise de celle-ci a influencé son choix d'appartenance : «L'aspect breton, pas vraiment. Pendant mon enfance, ce n'était pas forcément très positif de se sentir Breton : l'image de la Bretagne, les fest-noz, les bretonnants, les ploucs. Puis pendant mon adolescence, je vivais dans un milieu pas mal anglophone et je ne me sentais pas gallo ou breton. Mais je pense que l'on peut avoir une identité à plusieurs niveaux ».

Pour ces figures, il n'y a donc pas «d'ici » véritable ou durable : tout ici n'est qu'un ailleurs en sursis (Sencébé 2004 : 25). Zina (56 ans, émigrée en 1975) se situe dans le même état d'esprit en soulignant

19. En référence au Pays gallo en Haute-Bretagne, la partie orientale de la Bretagne. 
qu'elle perçoit sa migration comme perpétuelle et non définitive. Il n'y a donc ni perspective de retour en territoire d'origine, ni d'installation définitive en territoire d'accueil. Elle se situe dans un entre-deux, une situation transnationale dont elle essaie de tirer la meilleure expérience.

\begin{abstract}
Quand on a émigré, on n'avait pas acheté de billet de retour, même si je ne connaissais pas le pays. On ne savait pas où on irait ensuite. Ce n'était peut-être pas le pays idéal, mais c'était comme ça. Ça m’a donné la possibilité de travailler en français, même si j'étais capable de travailler en anglais, ça ne me dérangeait pas. Mon mari ne parlant pas anglais, si j'avais eu la possibilité, je serais peut-être allée ailleurs. On était internationaux, déjà. On se sentait comme appartenant au monde, c'est tout. On n'était pas cantonnés à un pays. On avait l'argent pour se payer les billets d'avions, ce que les gens ne pouvaient pas faire avant.
\end{abstract}

En effet, Helly et al. expliquent que « la multiplication et la facilité technique des échanges entre pays (informations, capital, déplacements, produits culturels) offrent désormais d'autres possibilités aux migrants, dont celles de demeurer en contact avec leur pays d'origine et d'en connaître l'évolution, comme de développer des identifications nouvelles, transnationales ou diasporiques » (2001:3). Comme le décrit Sencébé, avec ces nouvelles identifications, «les lieux et les liens sont ainsi substituables, mais non équivalents : ceux où l'on est situé ici et maintenant sont les plus intéressants après comparaison avec d'autres (non-équivalence), mais si une opportunité se présente on pourra aller ailleurs et avec d'autres (substituabilité)»(2004:25). Selon Helly et al. encore, cette figure est bel et bien celle du transnational, nomade, jouant de diverses affiliations pour mieux circuler dans le monde, se promouvoir, celle qui vit des appartenances arrimées à des fonctions économiques utilitaires et à des principes de liberté. Ces migrants se sentent "hors lieux ", comme le décrit une de mes interlocutrices. Pour eux, leur origine bretonne est donc surtout symbolique. Ils sont plutôt dans une démarche d'adaptabilité à leur territoire d'accueil.

\title{
Conclusion
}

Durant quatre ans, par le biais de cette recherche, j'ai essayé de révéler la manière dont certains Bretons en situation migratoire au Québec expriment, valorisent, voire réinventent ou non leur appartenance culturelle régionale bretonne, autrement dit leur 
bretonnité, à $6000 \mathrm{~km}$ de leur territoire d'origine. D'après les entretiens que j'ai menés, nombre de Bretons demeurant au Québec se sentent et se revendiquent Bretons plutôt que Français. Autrement dit, ils mobilisent la mémoire collective de leur territoire local d'origine pour exprimer leur appartenance culturelle régionale en terre d'accueil.

Certains de mes interlocuteurs ont réellement choisi leur bretonnité puisque leurs parents ou grands-parents refusaient de la leur transmettre, tandis que d'autres l'ont reçue en héritage et l'ont reproduite ou modelée en fonction du contexte dans lequel ils ont évolué. En effet, nous avons pu constater que pour certains, cet attachement à la Bretagne n'est pas linéaire, mais a fluctué avec le temps, l'âge et les expériences en territoire d'origine et d'accueil. Certains revendiquent leur bretonnité à un moment et pas à un autre en fonction de l'image positive ou négative qui leur a été transmise et de leurs besoins identitaires. Certains ont même redécouvert leur attachement à une appartenance culturelle bretonne seulement en territoire d'accueil. La déterritorialisation de ces Bretons n'a donc pas été synonyme de perte d'identité, bien au contraire. Comme l'expliquent Helly et al., "l'immigration est [...] toujours sous-tendue par la volonté de maintenir le sens d'une continuité, selon une dynamique fondamentale de toute identification personnelle et de toute recomposition culturelle. Et le désir de reproduire certaines pratiques, valeurs et mémoires familiales, sociales, voire nationales, constitue-t-il un aspect de tout projet migratoire»(2001:2). Autrement dit, le contexte de la migration est souvent révélateur d'une affirmation identitaire. Parmi ces migrants, certains ont également choisi la multi-appartenance bretonne, française, québécoise, voire montréalaise, car les appartenances à ces groupes leur procurent une certaine satisfaction. Enfin d'autres se déclarent « citoyens du monde », se sentant tels des " oiseaux migrateurs ", volant de territoire en territoire et se posant là où ils en tirent les meilleurs avantages. En réalité, une appartenance culturelle est toujours à construire. Elle n'est jamais figée. Elle n'est pas attachée aux seules « racines », mais se construit en fonction de la manière dont les individus en prennent conscience et se la réapproprient.

L'analyse de ces différentes postures m'a aidé à comprendre dans quelle mesure la nature des relations à un territoire d'origine et à un territoire d'accueil influence la prégnance d'une appartenance culturelle. Au cours de cette recherche, j'ai éclairé les formes d'appartenance culturelle de la communauté bretonne en situation de mobilité 
géographique. Mais mon ambition est d'appliquer cette recherche à d'autres cas d'étude, c'est-à-dire d'autres communautés culturelles, et ainsi de participer à l'enrichissement des connaissances dans les champs de l'anthropologie et de l'ethnologie des migrations. En effet, les phénomènes observés ne sont ni propres à la migration des Bretons, ni à celle vers le Québec en particulier, mais sont identifiables dans la plupart des populations en situation de migration. Cette étude de cas des Bretons au Québec est donc surtout un outil pour mieux comprendre les liens que maintiennent ou construisent les populations migrantes avec leur territoire d'origine et leur territoire d'accueil.

D'autre part, ces quatre années de doctorat m'ont conduite à vouloir explorer d'autres questionnements, notamment ceux relatifs à la transmission de cette appartenance culturelle bretonne des migrants de première génération aux migrants dits de seconde génération. Dans ce cadre, il serait particulièrement intéressant d'analyser le discours tenu par mes interlocuteurs sur les modes de transmissions de cette appartenance culturelle bretonne qu'ils ont mis en œuvre au Québec, ainsi que les discours tenus par les descendants de ces migrants afin de rendre compte éventuellement de la dimension transgénérationnelle de cette bretonnité en terre d'accueil. Mais ces données pourront être exploitées dans la perspective d'un travail de recherche prolongé ou d'une publication. 


\section{Références}

Appadurai, Arjun, 2001, Après le colonialisme. Les conséquences culturelles de la globalisation. Paris, Payot.

Bertaux, Daniel, 1980, «L'approche biographique. Sa validité méthodologique, ses potentialités ». Cahiers internationaux de sociologie 27 (69) : 197-225.

Broudig, Fanch, 1995, La Pratique du breton de l'Ancien Régime à nos jours. Rennes, Presses Universitaires Rennes II.

Butler, Kim D., 2001, «Defining Diaspora, Refining a Discourse ». Diaspora 10 (2) : 189-220.

Croix, Alain, 2001, «Prénoms », dans Alain Croix et Jean-Yves Veillard (dir.), Dictionnaire du patrimoine breton. Brest, Apogée.

Denzin, Norman K. et Yvonna S. Lincol, 2003, Collecting and Interpreting Qualitative Materials. Thousand Oaks, CA, Sage publications.

Dubois-Ouellet, Simonne, 1994, "Le récit de vie comme outil d'enquête. Expériences de terrain ». Canadian Folklore canadien 16 (1) : 55-72.

Fardon, Richard, 1995, «Introduction» dans Richard Fardon (dir.), Counterworks: Managing the Diversity of Knowledge. Londres et NewYork, Routledge.

Fischer, Michael M.J., 1991, « The Uses of Life Histories ». Anthropology and humanism 16 (1): 24-27.

Ghasarian, Christian (dir.), 2002, De l'ethnologie à l'anthropologie réflexive. Nouveaux terrains, nouvelles pratiques, nouveaux enjeux. Paris, Colin. Gonidec, Kristian, 1979, «Louis Bizien, de Laz, président des Bretons de Montréal ». Le Télégramme (Sud Finistère), 8 août : 5.

Green, Nancy L., 2002, Repenser les migrations. Paris, Presses Universitaires de France.

Helly, Denise, Michèle Vatz-Laaroussi et Lilyane Rachedi, 2001, "Transmission culturelle aux enfants par de jeunes couples migrants. Montréal, Québec, Sherbrooke ». Centre métropolis du Québec. [En ligne], http://im.metropolis.net/research-policy/ research content/doc/Transmission.pdf, consulté le 4 août 2011.

Isajiw, Wsevolod W., 1999, Understanding Diversity: Ethnicity and Race in the Canadian Context. Toronto, Thompson Educational Pub.

Lipiansky, Edmond Mark, 1995, «Communication interculturelle et modèles identitaires », dans Jean-Pierre Saez (dir.), Identités, cultures et territoires : 35-40. Paris, Desclée de Brouwer.

Maalouf, Amin, 1998, Les identités meurtrières. Paris, Grasset et Fasquelle. 
Marcus, Georges. E, 1998, "Ethnography in / of the World System: The Emergence of Multi-Sited Ethnography » dans Georges E. Marcus (dir.), Ethnography through Thick and Thin. Princeton, Princeton University Press, 79-104.

Mathieu, Jacques (dir.), 1983, Travaux et inédits d'Elli Kaija KöngäsMaranda. Cahiers du CÉLAT, 1, Québec, Université Laval.

Mimeault, Mario, 1999, «Les Bretons » dans Paul Robert Magocsi (dir.), Encyclopedia of Canada's Peoples : 282-287. Toronto, Buffalo, London, University of Toronto Press.

Ministère de l'immigration et des communautés culturelles (MICC), Direction de la recherche et de l'analyse prospective, 2005, Portrait statistique de la population immigrante, née en France, recensée au Québec en 2001. Compilation spéciale du MICC.

Monsutti, Alessandro, 2005, «En suivant les réseaux de Kaboul à NewYork: Quelques réflexions méthodologiques sur la recherche ethnographique parmi les migrants ». Ethnologies, vol. 27, n 1 : 3437.

Oury, Jean, 1989, Création et schizophrénie. Paris, Galilée.

Poirier, Jean, Simone Claper-Valladon et Paul Raybaut, 1983, Les récits de vie. Théorie et pratique. Paris, Presses universitaires de France.

Rosental, Paul-André, 1990, «Maintien/rupture. Un nouveau couple pour l'analyse des migrations». Annales ESC 45 (6) : 1407-1410. Saint-Martin, Fernande, 1992, «L'espace comme localisation et identification » dans Bogumil Jewsiewicki et Jocelyn Létourneau (dir.), Construction identitaire. Questionnements théoriques et études de cas : 127-137. Québec, CELAT, Université Laval.

Sayad, Abdelmalek, 1986, "La "vacance" comme pathologie de la contradiction de l'immigré. Le cas de la retraite et de la préretraite ", Gérontologie $60: 37-55$.

Sencébé, Yannick, 2004, «Être ici, être d'ici. Formes d'appartenance dans le Diois (Dôme)», Ethnologie française 34 (1) : 23-29.

Shiose, Yuki et Jacques Zylberberg, 1998, «L'identité culturelle et l'état d'anthropologue », dans Laurier Turgeon, Jocelyn Létourneau et Khadiyatoulah Fall (dir.), Les espaces de l'identité. Sainte-Foy, Québec, Presses de l'Université Laval ; Paris, Montréal, L'Harmattan,

Tajfel, Henri, 1978, « Social Categorization, Social Identity and Social Comparison » dans Henri Tajfel (dir.), Differenciation Between Social Groups. London, Academic Press.

Tardieu, Marc, 2003, Les Bretons à Paris. De 1900 à nos jours. Paris, Editions du Rocher. 
Texier, Marcel, 2004, La Bretagne n'a pas dit son dernier mot. Brest, Yoran embanner.

Turgeon, Laurier, 2003, Patrimoines métissés. Contextes coloniaux et postcoloniaux. Paris, Éditions de la Maison des sciences de l'Homme ; Québec, Les Presses de l'Université Laval.

Wolcott, Harry F. (1995). The Art of Fieldwork. Lanham, MN, Altamira Press. 